\title{
SISTEMA AUTOMÁTICO DE CLASSIFICAÇÃO DE DISTÚRBIOS ELÉTRICOS MÚLTIPLOS E ISOLADOS
}

\author{
Danton Diego Ferreira* \\ danton@deg.ufla.br \\ Carlos Augusto Duque ${ }^{\dagger}$ \\ carlos.duque@ufjf.edu.br
}

\author{
Augusto Santiago Cerqueira ${ }^{\dagger}$ \\ augusto. santiago@ufjf.edu.br
}

José Manoel de Seixas

seixas@lps.ufrj.br

\author{
Moisés Vidal Ribeiro ${ }^{\dagger}$ \\ moviribeiro@ieee.org \\ *Universidade Federal de Lavras \\ Departamento de Engenharia \\ Lavras, MG, Brasil \\ ${ }^{\dagger}$ Universidade Federal de Juiz de Fora \\ Laboratório de Sinais e Telecomunicações \\ Juiz de Fora, MG, Brasil \\ ${ }^{\ddagger}$ Universidade Federal do Rio de Janeiro \\ Laboratório de Processamento de Sinais, COPPE/Poli \\ Rio de Janeiro, RJ, Brasil
}

\begin{abstract}
Automatic System For Classification of Isolated and Multiple Disturbances in Electric Signals In this work, an automatic system was designed for classifying single and multiple disturbances in power systems. A filter bank was used for signal decomposition, which allows the decoupling of some disturbance classes, and makes it simpler the classifier design. The proposed system was designed using simulated data and tested for both simulated and experimental data. A global classification efficiency above $99 \%$ was achieved for the data set considered.
\end{abstract}

KEYWORDS: Power Quality, Multiple Disturbances, Filter Bank.

Artigo submetido em 06/11/2009 (Id.: 01073)

Revisado em 23/02/2010, 11/06/2010

Aceito sob recomendação do Editor Associado Prof. Eduardo N. Asada

\section{RESUMO}

Neste trabalho, um sistema de classificação de distúrbios elétricos múltiplos e isolados é proposto. A inovação deste sistema está na utilização de um conjunto de filtros no pré-processamento, o que possibilita a separação de algumas classes de distúrbios e, consequentemente, a classificação de distúrbios múltiplos a partir de classificadores mais simples. O sistema proposto foi projetado com dados simulados e testado tanto com dados simulados quanto com dados reais. Bons resultados foram alcançados, uma vez que um desempenho global superior a $99 \%$ foi obtido, para o conjunto de dados considerado.

PALAVRAS-CHAVE: Qualidade da Energia Elétrica, Múltiplos Distúrbios, Banco de Filtros. 


\section{INTRODUÇÃO}

O termo "qualidade de energia elétrica" (QEE), em geral, compreende a qualidade das formas de onda da tensão e corrente elétricas em um sistema elétrico (Bollen et al., 2009). Nas últimas duas décadas, este termo tem recebido bastante atenção de pesquisadores. Alguns dos principais fatores que fazem da QEE um tema de grande interesse são:

- O crescente uso de equipamentos computadorizados e sensíveis às perturbações do sistema elétrico;

- A exigência, por parte dos consumidores, de uma energia de melhor qualidade;

- O aumento do número de cargas de natureza nãolinear (sistemas microprocessados, fontes chaveadas, etc) em instalações elétricas residenciais, comerciais e industriais, o que provoca o aumento significativo da ocorrência de deformidades nas formas de onda da tensão e corrente (distúrbios) nos seus sistemas de distribuição;

- A regulamentação do setor elétrico, no que diz respeito à QEE, em fase de implantação.

O aparecimento de deformidades nas formas de onda da tensão e corrente caracterizam a falta de qualidade de energia elétrica. Estas deformidades são definidas pelo IEC (IEC-91) (110-R5, 1991) como fenômenos eletromagnéticos ou simplesmente distúrbios, dentre os quais destacam-se os harmônicos, interharmônicos, flutuações de tensão, afundamentos, transitórios, entre outros. Algumas modificações e complementações na classificação do IEC foram realizadas pela norma IEEE (IEEE-95) (Quality, 1995) com a inclusão da categoria de ruído, de elevação de tensão e a definição da categoria de distorção de forma de onda de corrente alternada, que inclui as distorções harmônicas, interharmônicas e de componente contínua.

Os distúrbios estão relacionados com diferentes eventos nos sistemas de potência. Alguns destes são gerados pela operação normal dos sistemas, tais como chaveamento de banco de capacitores e entrada e saída de grandes cargas lineares ou não, enquanto outros estão relacionados com eventos não operacionais do sistema, como as descargas atmosféricas e as faltas na rede elétrica. O fato é que, independentemente da causa, estes distúrbios podem causar perdas consideráveis aos consumidores de energia elétrica, sendo as indústrias as mais afetadas, uma vez que tais distúrbios podem levar à interrupção de um processo de produção ou, até mesmo, à queima de algum equipamento.
No Brasil, existe um esforço, por parte do governo, para a regulamentação dos padrões da qualidade de energia elétrica nos níveis de transmissão e distribuição. Atualmente, encontra-se em fase de regulamentação o PRODIST: Procedimentos de Distribuição (ANEEL, 2008), que, no seu módulo 8, contém documentos regulatórios com alguns indicadores de desempenho e de qualidade dos serviços de energia elétrica.

Neste contexto, o principal interesse dos pesquisadores é detectar e identificar (classificar) tais distúrbios, correlacionando-os com as possíveis causas, de forma a diagnosticar o problema em tempo mínimo.

Devido à variedade de distúrbios, o problema de classificação apresenta-se bastante complexo. Diversos trabalhos têm sido desenvolvidos neste sentido, com diferentes técnicas de processamento de sinais e inteligência computacional sendo utilizadas (Bollen et al., 2009).

Os distúrbios elétricos podem aparecer de forma isolada, ou seja, apenas um distúrbio em um dado trecho do sinal, ou de forma múltipla, que caracteriza os múltiplos distúrbios, em que mais de um distúrbio aparece simultaneamente ou em sequência no mesmo trecho do sinal. Os distúrbios isolados são melhor caracterizados e, portanto, mais fáceis de serem classificados (Bollen et al., 2009). Por outro lado, os múltiplos distúrbios geram formas de ondas difíceis de serem identificadas, até mesmo na presença de um especialista (Styvaktakis, 2002). Assim, a maioria das técnicas desenvolvidas, até o presente momento, concentram-se na classificação de distúrbios isolados e, o uso das mesmas gera resultados contraditórios e não representativos, quando aplicadas aos múltiplos distúrbios. Consequentemente, o uso de uma solução para a identificação da fonte geradora do distúrbio, tal como proposto em Azam et al. (2004), se torna bastante comprometido.

É importante destacar que o foco deste trabalho não é a identificação da fonte do distúrbio, ou do evento que o causou, mas apresentar uma técnica adequada para a classificação de distúrbios múltiplos e, com isto, oferecer subsídios para futuras ferramentas de identificação de fontes de distúrbios.

Em Ferreira, Marques, Cerqueira, Duque e Ribeiro (2009) e Ferreira, Cerqueira, Duque e Ribeiro (2009), foram propostos, respectivamente, sistemas de detecção e classificação de distúrbios elétricos utilizando parâmetros baseados em estatística de ordem superior (EOS) (Nikias e Petropulu, 1993). Ambos os trabalhos apresentaram resultados de desempenho próximos a $100 \%$ para o conjunto de dados considerado, porém, apenas distúrbios isolados foram abordados. 
Em Mishra et al. (2008), a transformada S (Stockwell et al., 1996) foi utilizada para extrair parâmetros juntamente com uma rede neural probabilística (Specht, 1990), que realiza a classificação. Os autores apresentam dez classes de distúrbios, sendo duas delas, distúrbios simultâneos de afundamentos de tensão com harmônicos e elevações de tensão com harmônicos. Uma eficiência global de $93,2 \%$ foi obtida para dados simulados em situação ruidosa.

Em Gargoom et al. (2008), a transformada S multiresolução (Chilukuri e Dash, 2004) e o teorema de Parseval (Proakis e Manolakis, 2006) foram utilizados para o monitoramento e a classificação de afundamentos e elevações de tensão, interrupções, transitórios oscilatórios e harmônicos. Informações tais como magnitude, frequências e período de duração dos distúrbios foram também disponibilizadas pelo algoritmo proposto. Os resultados foram apresentados na forma de estudos de caso utilizando-se software específico.

Biswal et al. (2009) propuseram um sistema híbrido, baseado no algoritmo fuzzy $C$-means e na técnica adaptive particle swarm optimization (APSO) (Ching-Yi e Fun, 2004). Nove classes de distúrbios foram abordadas, dentre as quais quatro de distúrbios simultâneos: flutuação de tensão com harmônicos, interrupção com harmônicos, afundamentos e elevações de tensão com harmônicos. O desempenho do método para cada classe de distúrbio variou entre $88 \%$ e $100 \%$.

Este trabalho propõe um sistema de classificação de distúrbios, considerando a possibilidade da ocorrência de doze classes de múltiplos distúrbios e seis classes de distúrbios isolados. Este sistema tem como principal característica a utilização de um banco de filtros com a função de desacoplar os múltiplos distúrbios. Um estudo sobre a complexidade computacional do sistema proposto, com vistas a uma futura implementação em tempo real, é também apresentado.

Este trabalho está organizando da seguinte maneira. A Seção 2 formula o problema de qualidade de energia elétrica e a Seção 3 desenvolve o sistema de classificação proposto. Na Seção 4, a metodologia aplicada para a obtenção dos resultados, que são mostrados na Seção 5 , é apresentada. Finalmente, na Seção 6, apresentamos as conclusões do trabalho .

\section{QUALIDADE DE ENERGIA ELÉTRICA}

Um sistema desenvolvido para a classificação de distúrbios de QEE deve identificar, corretamente, a ocorrência de cada anormalidade em um sinal de tensão do sistema elétrico de potência. Um sinal de tensão discreto no tempo $\{x(n)\}$ pode ser expresso como uma contribuição aditiva de vários tipos de fenômenos (Ribeiro, 2005)

$$
s(n)=\left.x(t)\right|_{t=n T_{s}}:=f(n)+h(n)+t(n)+u(n)
$$

onde $n=0, \cdots, N-1, T_{s}=\frac{1}{f_{s}}$ é o período de amostragem, as sequências $\{f(n)\},\{h(n)\},\{t(n)\}$ e $\{u(n)\}$ representam o componente fundamental, harmônicos, transitórios, e ruído (com distribuição normal $\mathcal{N}\left(0, \sigma_{u}^{2}\right)$ e independente de $\{f(n)\},\{h(n)\}$ e $\left.\{t(n)\}\right)$, respectivamente. Cada um destes sinais pode ser definido como:

$$
\begin{aligned}
f(n):=A_{0}(n) \cos \left(2 \pi \frac{f_{0}(n)}{f_{s}} n+\theta_{0}(n)\right), \\
h(n):=\sum_{m=1}^{M} h_{m}(n), \\
t(n):=\sum_{k=1}^{K} t_{k}(n),
\end{aligned}
$$

sendo que, em $(2), A_{0}(n), f_{0}(n)$ e $\theta_{0}(n)$ representam a amplitude, frequência fundamental e fase do sinal de tensão, respectivamente. Em $(3), h_{m}(n)$ é o $m$-ésimo harmônico:

$$
h_{m}(n):=A_{m}(n) \cos \left(2 \pi m \frac{f_{0}(n)}{f_{s}} n+\theta_{m}(n)\right),
$$

Sendo $A_{m}(n)$ a amplitude e $\theta_{m}(n)$ a fase do $m$-ésimo harmônico. $\operatorname{Em}(4),\left\{t_{k}(n)\right\}$ é o $k$-ésimo sinal transitório, tal como transitório oscilatório, chaveamento de grandes cargas, etc.

\section{SISTEMA DE CLASSIFICAÇÃO}

O sistema aqui proposto para classificação de distúrbios de QEE pode ser dividido, basicamente, em três blocos, conforme mostrado na Figura 1.

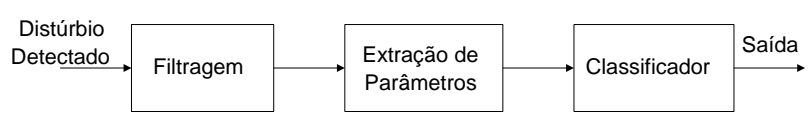

Figura 1: Diagrama em blocos simplificado do sistema de classificação proposto

O bloco de filtragem tem como objetivo desacoplar os múltiplos distúrbios, através da separação do sinal original em algumas faixas específicas de frequências. Após 
a filtragem, alguns parâmetros, que maximizam a fronteira de separação entre as diferentes classes de distúrbios, são extraídos com a finalidade de facilitar a tarefa de classificação, que é realizada no terceiro bloco.

É importante ressaltar que a excitação de entrada do classificador proposto está restrita a sinais de tensão contendo distúrbios, ou seja, é necessária a utilização de um bloco detector anterior, uma vez que o classificador proposto não incorpora a classe de sinais sem distúrbios. Para tal, o detector proposto em Ferreira, Marques, Cerqueira, Duque e Ribeiro (2009) foi utilizado, uma vez que apresenta um bom desempenho e uma reduzida complexidade computacional. Este detector utiliza EOS como parâmetros que alimentam um discriminador baseado no critério de Bayes (Duda et al., 2000). O discriminador classifica a janela de dados em duas categorias: com distúrbios e sem distúrbios. As janelas contendo distúrbios são, então, encaminhadas para a realização da classificação, através do diagrama mostrado na Figura 1.

\subsection{Filtragem}

A etapa de filtragem é constituída por quatro filtros IIR (Mitra, 2005), projetados de forma a possibilitar o desacoplamento dos diversos distúrbios a serem classificados. A idéia de utilizar filtros para desacoplar os múltiplos distúrbios parte da própria definição de que o sinal de tensão pode ser expresso como a contribuição de vários tipos de fenômenos, conforme mostrado na Seção 2.

A Figura 2 ilustra o diagrama em blocos em detalhes da etapa de filtragem. Inicialmente, um filtro Notch (Hirano et al., 1974), sintonizado na frequência do componente fundamental $(60 \mathrm{~Hz})$ do sinal monitorado, foi utilizado para dividir o sinal em dois componentes básicos, $e(n)$ e $\hat{f}(n)$. O sinal $\hat{f}(n)$, que corresponde à estimativa do componente fundamental do sinal de tensão monitorado, contém as informações do componente fundamental e dos distúrbios a ele relacionados, tais como afundamento e elevação de tensão. O componente $e(n)$, denominado aqui de componente de "erro", carrega as informações associadas aos distúrbios de frequências mais altas, tais como harmônicos, transitórios, impulsos, notches, entre outros.

A função de transferência do filtro Notch discreto de segunda ordem, com frequência de rejeição $w_{o}=2 \pi \frac{f_{o}}{f_{s}}$, pode ser vista em (6).

$$
H_{0}(z)=\frac{1+a_{0} z^{-1}+z^{-2}}{1+\rho_{0} a_{0} z^{-1}+\rho_{0}^{2} z^{-2}}
$$

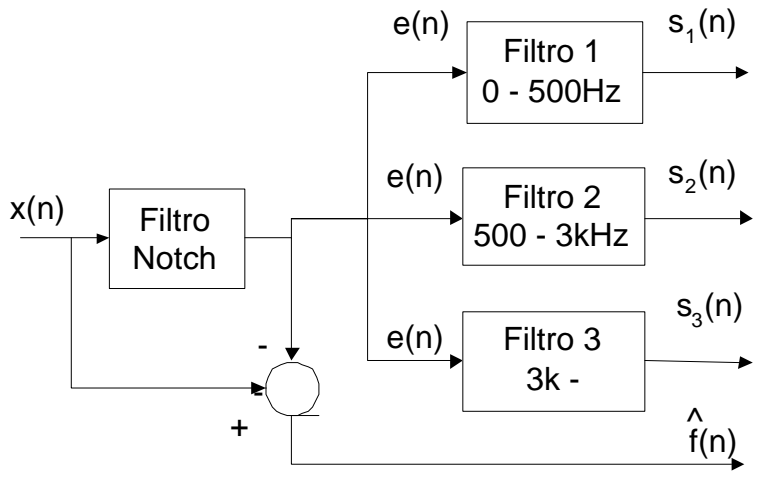

Figura 2: Diagrama em blocos do processo de filtragem de $x(n)$.

onde $a_{0}=-2 \cos \omega_{0}$ e $0 \ll \rho_{0}<1$ é o fator notch. Neste trabalho, utilizou-se $\rho_{0}=0,97$, que conjuga boa seletividade em frequência com um transitório não muito longo considerando-se a aplicação.

Com o objetivo de desacoplar os distúrbios presentes no sinal $\{e(n)\}$, mais três filtros foram utilizados. Para o projeto destes filtros, a informação espectral foi obtida da Transformada Discreta de Fourier (TDF) (Mitra, 2005) dos distúrbios presentes no sinal $\{e(n)\}$. A Figura 3 mostra a média dos módulos das transformadas para 200 eventos de transitório oscilatório, harmônicos, notch e impulsos. Estas informações espectrais evidenciam a faixa de frequência onde há maior concentração de energia referente a estes distúrbios. Assim, projetou-se um filtro passa-baixa com frequência de corte de $500 \mathrm{~Hz}$, buscando eventos de baixa frequência, um filtro passa-faixa com frequências de corte em $500 \mathrm{~Hz}$ e $3 \mathrm{kHz}$, buscando eventos de média frequência, e um filtro passa-alta com frequência de corte de $3 \mathrm{kHz}$, buscando eventos de alta frequência. Na saída do filtro passa-baixa, os harmônicos e impulsos aparecem com energia relevante. Na saída do filtro passafaixa, os distúrbios de transitório oscilatórios, notches e harmônicos aparecem com maior energia. A saída do filtro passa-alta fica restrita, basicamente, ao aparecimento de notches.

Os três filtros projetados são IIR de quarta ordem e foram desenvolvidos utilizando aproximação elíptica (Mitra, 2005). A Equação (7) mostra a função de transferência de um filtro IIR de quarta ordem.

$$
H(z)=\frac{b_{0}+b_{1} z^{-1}+\cdots+b_{4} z^{-4}}{a_{0}+a_{1} z^{-1}+\cdots+a_{4} z^{-4}}
$$

Neste ponto, é importante tecer alguns comentários sobre a distribuição espectral para os harmônicos, mos- 


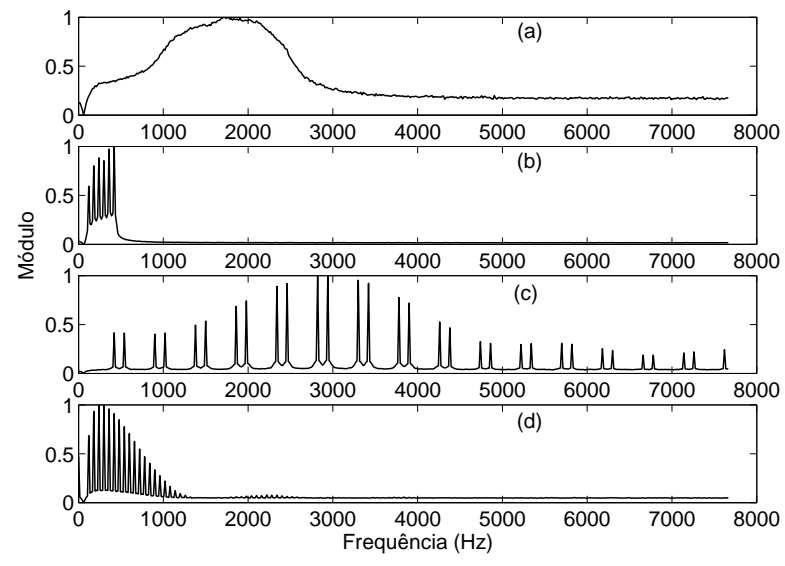

Figura 3: Módulo médio normalizado da TDF aplicada aos sinais $e(n)$ das classes: (a) Transitório oscilatório, (b) Harmônico, (c) Notch e (d) Impulsos.

trada na Figura 3(b). Nota-se claramente uma concentração de energia para as frequências menores que $1 \mathrm{kHz}$. Num primeiro momento pode parecer contraditório, pois muitas normas estabelecem a medição de harmônicos de ordem elevada. Por exemplo, o PRODIST estabelece até o $25^{\circ}$ componente (ANEEL, 2008). Entretanto, duas observações justificam a faixa espectral utilizada: (1) Os componentes harmônicos, presentes no sinal de tensão, devem apresentar energias decrescentes, com o aumento da frequência. As mesmas normas estabelecem o máximo de distorção harmônica individual (energia) para cada componente harmônico (ANEEL, 2008), o que foi levado em conta para gerar o banco de dados, que incluiu componentes até a $7^{\mathrm{a}}$ ordem; (2) A técnica de separação por filtros utilizada neste trabalho procura contribuir para a melhor separação das classes, o que possibilita o projeto de classificadores mais eficientes, porém, em toda técnica de separação poderá existir alguma interseção entre as classes.

\subsection{Extração de Parâmetros}

Após a filtragem do sinal $\{x(n)\}$ e a obtenção dos sinais $\{\hat{f}(n)\},\left\{s_{1}(n)\right\},\left\{s_{2}(n)\right\}$ e $\left\{s_{3}(n)\right\}$ (veja a Fig. 2), alguns parâmetros são extraídos, visando à redução de dimensionalidade da informaçao e um melhor desempenho na classificação. A Figura 4 mostra o diagrama em blocos da extração de parâmetros.

Para o sinal $\left\{s_{3}(n)\right\}$, que carrega a informação de notches, o valor RMS (Root Mean Square) foi utilizado, já que, para os eventos considerados, apenas ruído e eventos de notch têm componentes espectrais acima de $3 \mathrm{kHz}$
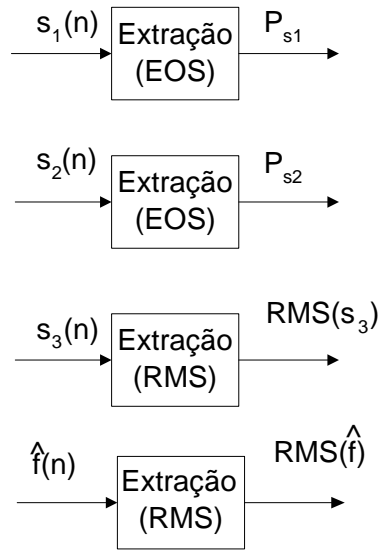

Figura 4: Diagrama em blocos do processo de extração de parâmetros.

e, portanto, o parâmetro RMS pode ser utilizado para separar o notch dos demais distúrbios. O valor RMS é calculado da seguinte forma:

$$
R M S(y)=\sqrt{\frac{\sum_{n=1}^{N}(y(n)-\bar{y})^{2}}{N-1}}
$$

onde $N$ é o número de amostras do sinal discreto no tempo $\{y(n)\}$ e $\bar{y}$ é a sua média aritmética. O valor RMS foi também utilizado sobre o sinal $\{\hat{f}(n)\}$, com o objetivo de separar afundamentos de elevações de tensão.

Os sinais $\left\{s_{1}(n)\right\}$ e $\left\{s_{2}(n)\right\}$ podem representar mais de uma classe de distúrbios e, portanto, o uso de uma extração de parâmetros mais elaborada, de forma a maximizar as fronteiras de separação entre estes distúrbios, foi utilizada. Neste caso, parâmetros baseados em EOS (Mendel, 1991), especificamente, os cumulantes de segunda e quarta ordem foram utilizados. O uso de cumulantes como parâmetros para a classificação de distúrbios foi inicialmente proposta, com bastante sucesso, em Ribeiro (2005). As vantagens do uso de cumulantes, como parâmetros para caracterizar os distúrbios, são: (i) carregam informações importantes para a classificação dos mesmos e (ii) os cumulantes de ordem maior que 2 são nulos para ruídos gaussianos, o que torna o sistema imune a estes ruídos.

Os cumulantes de $2^{\mathrm{a}}$ e $4^{\mathrm{a}}$ ordens, para um vetor $\mathbf{z}$ constituído de variáveis aleatórias, e de comprimento $N$ finito, podem ser estimados, como demonstrado em Ribeiro et al. (2006), por: 


$$
\hat{c}_{2, z}(i):=\frac{1}{N} \sum_{n=0}^{N-1} z(n) z[\bmod (n+i, N)],
$$

$$
\begin{gathered}
\hat{c}_{4, z}(i):=\frac{1}{N} \sum_{n=0}^{N-1} z(n) z^{3}[\bmod (n+i, N)]- \\
\frac{1}{N^{2}} \sum_{n=0}^{N-1} z(n) z[\bmod (n+i, N)] \sum_{n=0}^{N-1} z^{2}(n)
\end{gathered}
$$

onde $\bmod (n+i, N)$ é o resto inteiro da divisão de $n+i$ por $N$.

Uma vez que os cumulantes tenham sido extraídos, deve-se selecionar, aqueles mais representativos de cada classe, ou seja, os que permitem obter-se uma maior fronteira de separação entre as classes. Assim, decidiu-se usar o FDR (Fisher Discriminat Ratio) pela sua simplicidade e obtenção de resultados satisfatórios (Theodoridis e Koutroumbas, 2009). A função custo do critério FDR é:

$$
\mathbf{J}_{c}=\left(\mathbf{m}_{1}-\mathbf{m}_{2}\right)^{2} \odot \frac{1}{\mathbf{D}_{1}^{2}+\mathbf{D}_{2}^{2}}
$$

onde $\mathbf{J}_{c}=\left[J_{1} \cdots J_{L_{l}}\right]^{T}, \quad L_{l}$ é o número total de parâmetros, $\mathbf{m}_{1}$ e $\mathbf{m}_{2}$, e $\mathbf{D}_{1}^{2}$ e $\mathbf{D}_{2}^{2}$ são, respectivamente, os vetores de média e variância dos vetores de parâmetros das classes 1 e 2 . O símbolo $\odot$ refere-se ao produto de Hadamard $\mathbf{r} \odot \mathbf{s}=\left[r_{0} s_{0} \cdots r_{L_{r}-1} s_{L_{r}-1}\right]^{T}$. O $i$-ésimo elemento do vetor de parâmetros dado por (11) com o maior valor é selecionado para ser usado pelo classificador. Aplicando este procedimento para todos os elementos do vetor de parâmetros principal, os $K$ parâmetros associados com os $K$ maiores valores no vetor $\mathbf{J}_{\mathbf{c}}$ são usados pelo classificador.

Após a seleção dos cumulantes, são obtidos os vetores de parâmetros $\left(\mathbf{P}_{s 1}\right.$ e $\left.\mathbf{P}_{s 2}\right)$ relativos aos sinais $\left\{s_{1}(n)\right\}$ e $\left\{s_{2}(n)\right\}$, respectivamente, conforme mostra a Figura 4 . Cada vetor é composto por 16 parâmetros, sendo $8 \mathrm{cu}-$ mulantes de segunda ordem e 8 de quarta ordem. O número de parâmetros foi escolhido na fase de treinamento do classificador, de forma a maximizar o desempenho do mesmo.

\subsection{Etapa de Classificação}

$\mathrm{Na}$ etapa de classificação baseada na análise do sinal de erro $\{e(n)\}$, utilizam-se $\mathbf{P}_{s 1}, \mathbf{P}_{s 2}$ e $R M S\left(s_{3}\right)$, que são os vetores de parâmetros extraídos dos sinais $\left\{s_{1}(n)\right\}$, $\left\{s_{2}(n)\right\}$ e $\left\{s_{3}(n)\right\}$, respectivamente, sendo que o último é composto por um único parâmetro, o valor $R M S$ do sinal $\left\{s_{3}(n)\right\}$. Na etapa referente à análise do sinal $\{\hat{f}(n)\}$, utiliza-se o valor $R M S$ do sinal $\{\hat{f}(n)\}$ como único parâmetro.

Quatro classificadores independentes são utilizados para classificar os distúrbios associados ao sinal $\{x(n)\}$, conforme mostra a Figura 5. O bloco Decisor Classe implementa o algoritmo que decide sobre a ocorrência das diferentes classes de distúrbios que aparecem no sinal monitorado.

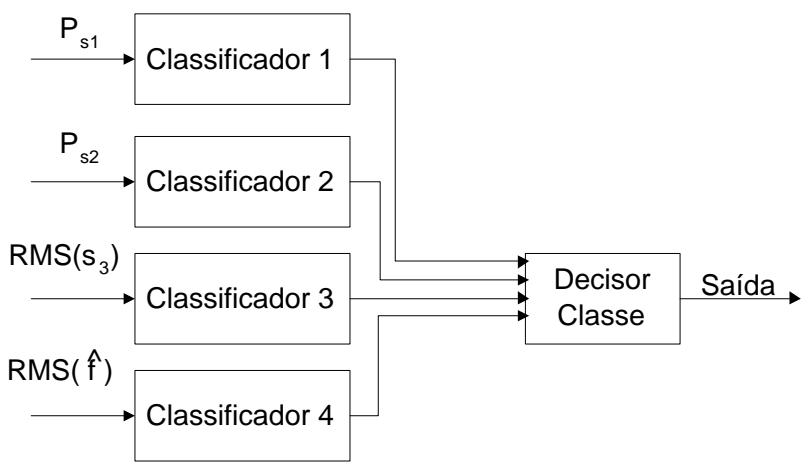

Figura 5: Diagrama em blocos do Classificador Final

Os blocos Classificador 1 e Classificador 2 são constituídos por uma rede neural artificial (RNA) de três camadas (Haykin, 2008), de modo a explorar a informação EOS. O bloco Classificador 3 é constituído por uma fronteira linear, de modo que valores de $R M S\left(s_{3}\right)$ acima de um patamar $(\alpha)$, previamente definido com dados de treinamento, indicam distúrbios de notches. O Classificador 4 é constituído por duas fronteiras lineares, objetivando separar 3 classes: afundamentos de tensão, elevações de tensão e sinais com o componente fundamental sem alterações. Neste caso, dois patamares são previamente definidos, $\beta 1$ e $\beta 2$. Logo, se $R M S(\hat{f})<\beta 1$, classifica-se o distúrbio como afundamento de tensão. Se $\beta 1<R M S(\hat{f})<\beta 2$, sinais são considerados com o componente fundamental sem alterações e, caso $R M S(\hat{f})>\beta 2$, indentifica-se uma elevação de tensão.

As RNAs utilizadas foram compostas por 16 nós de entrada, referentes aos 8 cumulantes de segunda e quarta ordem selecionados utilizando o critério de maior FDR, 10 neurônios na única camada oculta e quatro nós de saída. Os nós de saída são associados, na rede referente ao classificador 1 , às classes de harmônicos, impulsos, afundamentos/elevações de tensão e sinais sem estes distúrbios. Já os nós de saída da rede neural referente ao classificador 2 representam as classes de notches, impulsos, transitório oscilatório e sinais sem estes distúrbios. O nó de saída que apresentar o maior valor define a qual classe de distúrbio o evento pertence. 
De fato, os quatro classificadores abordam algumas classes de distúrbios em comum, como os classificadores 2 e 3, ambos utilizados para a identificação da classe de notches. Neste caso, como o classificador 3 é especialista em notches, sua saída terá prioridade quanto à afirmação da ocorrência ou não do notch pelo sistema classificador. Para isso, o bloco Decisor Classe implementa uma lógica, baseada na saída dos classificadores, que decide qual ou quais distúrbios apareceram no trecho do sinal de tensão em análise, resultando na saída final do classificador.

\section{METODOLOGIA}

Neste trabalho, foram abordadas seis classes de distúrbios isolados, oito classes com ocorrência simultânea (múltiplos distúrbios) envolvendo dois distúrbios e quatro classes de múltiplos distúrbios envolvendo três distúrbios. As classes são:

1. Harmônicos (S1);

2. Afundamento de tensão (S2);

3. Elevação de tensão (S3);

4. Transitório Oscilatório (S4);

5. Notch (S5);

6. Impulsos (S6);

7. Afundamento de tensão com harmônicos (S7);

8. Elevação de tensão com harmônicos (S8);

9. Afundamento de tensão com transitório oscilatório (S9);

10. Elevação de tensão com transitório oscilatório (S10);

11. Afundamento de tensão com notch (S11);

12. Elevação de tensão com notch (S12);

13. Notch com harmônicos (S13);

14. Transitório oscilatório com harmônicos (S14);

15. Afundamento de tensão com transitório oscilatório e harmônicos (S15);

16. Afundamento de tensão com notch e harmônicos (S16);

17. Elevação de tensão com notch e hamônicos (S17);

18. Elevação de tensão com transitório oscilatório e harmônicos (S18).
Para o projeto e validação do sistema proposto, foram utilizados 1.000 eventos simulados de cada classe de distúrbios (S1-S18). Estes sinais foram gerados sinteticamente com uma relação sinal ruído (SNR - Signal-toNoise Ratio) de $30 \mathrm{~dB}, N=1.024$ amostras, frequência de amostragem $f a=15.260 \mathrm{~Hz}$, seguindo as definições encontradas na norma do IEEE (Quality, 1995).

Com o objetivo de atingir um elevado índice de generalização no processo de classificação, as características de cada evento foram escolhidas de forma aleatória em intervalos pré-definidos. Dentro dos intervalos foi considerado que as características (início do distúrbio, intensidade, etc.) deveriam apresentar uma distribuição estatística uniforme, de maneira a evitar a ocorrência de um maior número de eventos com alguma característica semelhante.

Dos 1000 eventos gerados para cada classe, 200 foram usados para treinamento dos classificadores ${ }^{1}$, 300 para validação e 500 foram utilizados para teste.

Para avaliar a eficiência do sistema proposto em dados reais, duas formas de onda da tensão com ocorrências de múltiplos distúrbios, adquiridos pelo grupo de trabalho do IEEE (working group P1159.3), foram apresentadas ao sistema proposto. Estas formas de onda estão ilustradas na Figura 6. É importante ressaltar que estes sinais foram adquiridos com frequência de amostragem semelhante à frequência utilizada no projeto do classificador $(f a=15.260 \mathrm{~Hz})$ e SNR em torno de $40 \mathrm{~dB}$.
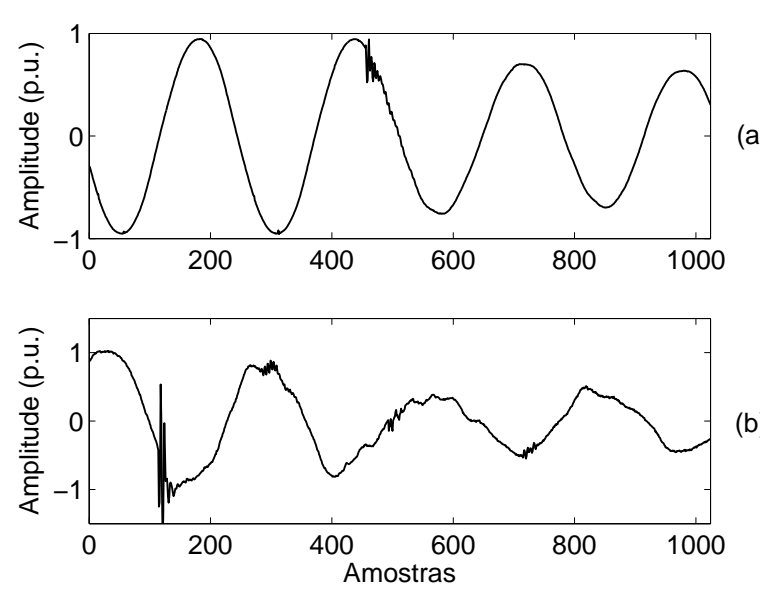

Figura 6: Formas de onda de aquisições reais do sinal de tensão com distúrbios múltiplos de transitórios oscilatórios e afundamento de tensão em (a) e transitórios oscilatórios, harmônicos e afundamento de tensão em (b)

\footnotetext{
${ }^{1}$ A Figura 3 foi gerada a partir dos eventos de treinamento.
} 


\section{RESULTADOS E DISCUSSÕES}

Esta seção apresenta os resultados e discussões do sistema proposto para as aplicações em dados de teste simulados e experimentais.

\subsection{Dados Simulados}

Os resultados finais do classificador, para os eventos de validação, são mostrados na Tabela 1.

Analisando a Tabela 1, pode-se observar que o desempenho na classificação para distúrbios isolados é muito bom, com a eficiência se situando acima de 99,0 \% (S1S6). Para distúrbios múltiplos (S7-S18), o desempenho cai levemente, com a eficiência de classificação ficando acima de $97,2 \%$. Uma eficiência global de 99,1\%, considerando-se a média aritmética dos desempenhos obtidos para cada classe de distúrbio, foi alcançada.

A Tabela 1 mostra a matriz de confusão do classificador, permitindo uma análise mais detalhada do seu desempenho. É importante verificar que, para os casos de múltiplos distúrbios, quando o classificador erra, o resultado é apontado para um dos distúrbios que compõe o múltiplo distúrbio analisado. Para as classes de múltiplos distúrbios compostas por dois distúrbios (S7S14), o percentual de erro do classificador sempre aponta para uma das classes que compõe o múltiplo distúrbio e, para as classes compostas por três distúrbios (S15-S18), o percentual de erro do classificador se concentra sempre em uma classe de dois distúrbios que faz parte da classe original (com três distúrbios). Portanto, nestes casos, pode-se dizer que o classificador acertou parcialmente a classificação, omitindo a ocorrência de um dos distúrbios que compõe o múltiplo distúrbio analisado.

Como os eventos de harmônicos, impulsos e notches são avaliados por mais de um classificador, a lógica final, implementada no bloco Decisor Classe (vide Fig. 5) bloqueia a saída dos classificadores com desempenho inferior para estas classes, deixando a classificação destes distúrbios restrita ao classificador com melhor desempenho. Isso explica o fato do erro de $1 \%$ para a classe S1 (vide Tab. 1) não aparecer em outras classes, ou seja, neste caso, o classificador apontou os eventos relacionados a este percentual de erro para uma classe cuja saída foi bloqueada pela lógica final.

\subsection{Dados Reais}

Nesta etapa, as formas de onda mostradas na Figura 6 foram utilizadas como exemplo de aplicação, conforme mencionado na Seção 4.
Pode-se verificar a presença de um leve transitório oscilatório de curta duração seguido de um afundamento de tensão na forma de onda da Figura 6 (a). O sistema proposto acertou a classificação deste sinal, apontandoo como um múltiplo distúrbio de transitório oscilatório com afundamento de tensão, classe S9, conforme esperado.

Na Figura 6 (b), pode-se observar a presença de um transitório oscilatório de curta duração, com uma intensidade superior ao da Figura 6 (a), seguido de afundamento de tensão, suaves transitórios e harmônicos. O sistema proposto, classifica este sinal, como pertencente à classe S15, referente a afundamento de tensão, com transitório oscilatório e harmônicos.

É importante ressaltar que o banco de dados reais do IEEE utilizado neste exemplo é composto de 60 formas de ondas com diferentes ocorrências de distúrbios. No entanto, não há uma classificação prévia destes e, portanto, as duas formas de ondas mostradas na Figura 6 foram escolhidas devido à sua fácil caracterização via inspeção visual.

Estes dois exemplos podem mostrar a boa capacidade do método para aplicações com dados reais.

\subsection{Complexidade Computacional}

A Tabela 2 mostra a complexidade computacional do método proposto, em termos do número de operações realizadas na fase de operação. O número de operações foi obtido conforme Tabela 3, em termos do número de amostras $(N)$ utilizadas, em que $M$ representa a ordem do filtro IIR utilizado. O número de entradas, saídas e neurônios na camada intermediária da rede neural utilizada é dado, respectivamente, por $N_{e}, N_{s}$ e $N_{n}$.

Dado o número de classes de distúrbios abordadas, incluindo os múltiplos distúrbios, é justificável que o sistema proposto apresente uma complexidade computacional razoável, conforme mostra a Tabela 2. Embora o foco do trabalho não seja a implementação em tempo real do sistema de classificação proposto, espera-se em uma etapa futura implementá-lo em tempo real utilizando processadores DSP (Digital Signal Processor) ou dispositivos FPGA (Field Program Gate Arrays).

\section{CONCLUSÕES}

Neste trabalho, um sistema para classificação de distúrbios elétricos em sistemas de potência, baseado na utilização de filtros para desacoplar múltiplos distúrbios, foi apresentado. A filtragem do sinal de distúrbio em di- 
Tabela 1: Matriz de confusão para a classificação relativa ao conjunto de teste.

\begin{tabular}{|c|c|c|c|c|c|c|c|c|c|c|c|c|c|c|c|c|c|c|}
\hline$(\%)$ & $\mathrm{S} 1$ & $\mathrm{~S} 2$ & S3 & S4 & S5 & S6 & S7 & S8 & S9 & S10 & S11 & $\mathrm{S} 12$ & $\mathrm{~S} 13$ & S14 & S15 & S16 & S17 & S18 \\
\hline S1 & 99,0 & & & & & & & & & & & & & & & & & \\
\hline S2 & 0,0 & 100 & & & & & & & & & & & & & & & & \\
\hline S3 & 0,0 & 0,0 & 100 & & & & & & & & & & & & & & & \\
\hline S4 & 1,0 & 0,0 & 0,0 & 99,0 & & & & & & & & & & & & & & \\
\hline S5 & 0,0 & 0,0 & 0,0 & 0,0 & 100 & & & & & & & & & & & & & \\
\hline S6 & 0,0 & 0,0 & $\overline{0,0}$ & 0,0 & 0,0 & 100 & & & & & & & & & & & & \\
\hline S7 & 0,2 & 0,8 & $\overline{0,0}$ & $\overline{0,0}$ & 0,0 & 0,0 & 99,0 & & & & & & & & & & & \\
\hline S8 & 0,0 & 0,0 & 2,8 & 0,0 & 0,0 & 0,0 & 0,0 & 97,2 & & & & & & & & & & \\
\hline S9 & 0,0 & 0,0 & 0,0 & 0,0 & 0,0 & 0,0 & 0,0 & 0,0 & 100 & & & & & & & & & \\
\hline S10 & $\overline{0,0}$ & $\overline{0,0}$ & $\overline{0,0}$ & 0,6 & 0,0 & $\overline{0,0}$ & $\overline{0,0}$ & $\overline{0,0}$ & 0,0 & 99,4 & & & & & & & & \\
\hline S11 & 0,0 & 0,0 & 0,0 & 0,0 & 0,0 & 0,0 & 0,0 & 0,0 & 0,0 & 0,0 & 100 & & & & & & & \\
\hline S12 & 0,0 & 0,0 & 0,0 & 0,0 & 1,0 & 0,0 & 0,0 & 0,0 & 0,0 & 0,0 & 0,0 & 99,0 & & & & & & \\
\hline S13 & 0,0 & 0,0 & 0,0 & 0,0 & 0,6 & 0,0 & 0,0 & 0,0 & 0,0 & 0,0 & 0,0 & 0,0 & 99,4 & & & & & \\
\hline S14 & 0,8 & 0,0 & 0,0 & 1,0 & 0,0 & 0,0 & 0,0 & 0,0 & 0,0 & 0,0 & 0,0 & 0,0 & 0,0 & 98,2 & & & & \\
\hline S15 & 0,0 & 0,0 & 0,0 & 0,0 & 0,0 & 0,0 & 0,6 & 0,0 & 1,2 & 0,0 & 0,0 & 0,0 & 0,0 & 0,0 & 98,2 & & & \\
\hline S16 & 0,0 & 0,0 & 0,0 & 0,0 & 0,0 & 0,0 & 0,0 & 0,0 & 0,0 & 0,0 & 1,2 & 0,0 & 0,4 & 0,0 & 0,0 & 98,4 & & \\
\hline S17 & 0,0 & 0,0 & 0,0 & 0,0 & 0,0 & 0,0 & 0,0 & 0,0 & 0,0 & 0,0 & 0,0 & 1,2 & 0,0 & 0,0 & 0,0 & 0,0 & 98,8 & \\
\hline S18 & 0,0 & 0,0 & 0,0 & 0,0 & 0,0 & 0,0 & 0,0 & 0,2 & 0,0 & 2,0 & 0,0 & 0,0 & 0,0 & 0,0 & 0,0 & 0,0 & 0,0 & 97,8 \\
\hline
\end{tabular}

Tabela 2: Complexidade computacional do método proposto.

\begin{tabular}{c||c} 
Operação & \\
\hline \hline Soma & 94.074 \\
\hline Multiplicação & 123.758 \\
\hline $\tan ()$. & 30
\end{tabular}

Tabela 3: Custo computacional das rotinas utilizadas no método proposto para classificação de múltiplos distúrbios.

\begin{tabular}{c||c|c|c} 
Rotinas & Somas & Multiplicações & $\tan ()$. \\
\hline \hline RMS & $3 N$ & $N+1$ & - \\
\hline Filtro Notch & $4 N$ & $3 N$ & - \\
\hline Filtro IIR & $N(2 M)$ & $N(2 M+1)$ & - \\
\hline $\begin{array}{c}\text { Cumulante de } \\
2^{a} \text { Ordem }\end{array}$ & $N-1$ & $N+1$ & - \\
\hline $\begin{array}{c}\text { Cumulante de } \\
4^{a} \text { Ordem }\end{array}$ & $3 N-2$ & $5(N+1)$ & - \\
\hline Rede Neural & $N_{n}\left(N_{e}+N_{S}+1\right)+N_{S}$ & $N_{n}\left(N_{e}+N_{s}\right)$ & $N_{n}+N_{S}$ \\
\hline Normalização & - & $N$ & -
\end{tabular}

ferentes bandas, associadas à ocorrência dos distúrbios, gerou quatro sinais, o que simplificou o projeto dos classificadores de distúrbios, possibilitando uma boa eficiência de classificação, mesmo com a ocorrência de múltiplos distúrbios.

A principal inovação no sistema proposto está na forma como os filtros foram projetados, com base na distribuição espectral dos distúrbios, resultando em apenas quatro filtros para separação do sinal. Com a separação parcial dos múltiplos distúrbios, proporcionada pelos filtros, foi possível projetar classificadores restritos a uma ou no máximo três classes de distúrbios, o que eleva a eficiência de classificação e diminui a complexidade do projeto. Assim, cruzando as informações das saídas de cada classificador através de uma lógica simples, foi possível abordar seis classes de distúrbios isolados e doze de múltiplos distúrbios com um bom desempenho e razoável complexidade computacional.

O sistema pode facilmente ser expandido para mais classes de múltiplos distúrbios (envolvendo os seis distúrbios isolados considerados) sem a necessidade de reprojetálo, bastando, para isso, apenas alterar a lógica final do sistema, incluindo a nova classe de múltiplo distúrbio. Também outros distúrbios, não apresentados neste trabalho, tais como flutuação de tensão e desequilíbrios podem ser acrescentados para formar novas combinações de distúrbios múltiplos a serem classificados. Estas novas classes serão alvos de trabalhos futuros.

Por fim, cumpre destacar a importância de se investigar classificadores para múltiplos distúrbios. Esta importância encontra-se no fato de que a identificação da fonte do distúrbio ou do evento que o causou poderá se beneficiar desta investigação. Este, também, torna-se um desafio para trabalhos futuros.

\section{AGRADECIMENTOS}

Agradecemos ao Conselho Nacional de Desenvolvimento Científico e Tecnológico (CNPq), à Coordenação e Aperfeiçoamento de Pessoal de Nível Superior (CAPES), à Fundação de Amparo à Pesquisa do Estado de Minas Gerais (FAPEMIG) e à Fundação de Amparo à Pesquisa do Estado do Rio de Janeiro (FAPERJ) pelo apoio a este projeto.

\section{REFERÊNCIAS}

110-R5, T. S. (1991). IEC-91- Draft Classification of Electromagnetic Environments, Technical report, IEC. 
ANEEL (2008). PRODIST: Procedimentos de Distribuição, www.aneel.gov.br .

Azam, M. S., Tu, F., Pattipati, K. R. e Karanam, R. (2004). A dependency model-based approach for identifying and evaluating power quality problems, IEEE Transaction on Power Delivery 19(3): 11541166 .

Biswal, B., Dash, P. K. e Panigrahi, B. K. (2009). Power Quality Disturbance Classification Using Fuzzy C-Means Algorithm and Adaptive Particle Swarm Optimization, IEEE Transactions on Industrial Electronics 56(1): 212-220.

Bollen, M. H. J., Ribeiro, P. F., Gu, I. Y. H. e Duque, C. A. (2009). Trends, challenges and opportunities in power quality research, EUROPEAN, Transactions on Electrical Power 20: 3-18.

Chilukuri, M. V. e Dash, P. K. (2004). Multiresolution S-transform-based fuzzy recognition system for power quality events, IEEE Transaction on Power Delivery 19(1): 323-330.

Ching-Yi, C. e Fun, Y. (2004). Particle swarm optimization algorithm and its application to clustering analysis, Proc. IEEE ICNSC pp. 789-794.

Duda, R. O., Hart, P. E. e Stork, D. G. (2000). Pattern Classification, 2 edn, John Wiley \& Sons.

Ferreira, D. D., Cerqueira, A. S., Duque, C. A. e Ribeiro, M. V. (2009). HOS-based method for classification of power quality disturbances, Electronics Letters 45(3): 183-185.

Ferreira, D. D., Marques, C. A. G., Cerqueira, A. S., Duque, C. A. e Ribeiro, M. V. (2009). Sistema Automático de Detecção e Classificação de Distúrbios Elétricos em Qualidade de Energia Elétrica, Sba: Controle \&3 Automação Sociedade Brasileira de Automatica 20(1): ISSN 0103-1759.

Gargoom, A. M., Ertugrul, N. e Soong, W. L. (2008). Automatic Classification and Characterization of Power Quality Events, IEEE Transactions on Power Delivery 23(4): 2417-2425.

Haykin, S. (2008). Neural networks and Learning Machines, 3 edn, Prentice Hall.

Hirano, K., Nishimura, S. e Mitra, S. K. (1974). Design of digital notch filters, IEEE Trans. on Communications 22(7): 964-970.
Mendel, J. M. (1991). Tutorial on higher-order statistics (spectra) in signal processing and system theory: theoretical results and some applications, Proc. of the IEEE 79(3): 278-305.

Mishra, S., Bhende, C. N. e Panigrahi, B. K. (2008). Detection and Classification of Power Quality Disturbances Using S-Transform and Probabilistic Neural Network, IEEE Transactions on Power Delivery 23(1): 280-280.

Mitra, S. K. (2005). Digital Signal Processing: A computer-based approach, 3 edn, McGraw-Hill.

Nikias, C. L. e Petropulu, A. P. (1993). Higher-Order Spectra Analysis - A Nonlinear Signal Processing Framework, Englewood Cliffs, Prentice Hall, NJ.

Proakis, J. G. e Manolakis, D. G. (2006). Digital Signal Processing, 4 edn, Prentice Hall.

Quality, I. S. C. C. . P. (1995). IEEE recommended practice for monitoring electric power quality, Technical report, IEEE.

Ribeiro, M. V. (2005). Técnicas de Processamento de Sinais Aplicadas à Trasmissão de Dados Via Rede Elétrica e ao Monitoramento da Qualidade de Energia, Dissertação Ph.D., Universidade de Campinas (UNICAMP).

Ribeiro, M. V., Marques, C. A. G., Duque, C. A., Cerqueira, A. S. e Pereira, J. L. R. (2006). Power Quality Disturbances Detection Using HOS, IEEE Power Engineering Society General Meeting p. 6. Monreal, QC, Canada.

Specht, D. F. (1990). Probabilistic neural networks, Neural Network 3(1): 109-118.

Stockwell, R. G., Mansinha, L. e Lowe, R. P. (1996). Localization of the complex spectrum: The Stransform, IEEE Transaction Signal Processing 44(4): 998-1001.

Styvaktakis, E. (2002). Automating Power Quality Analysis, Ph.D. dissertation, Dept. of Signals and Systems, Chalmers Univ. Technoloty, Gothenburg, Sweden.

Theodoridis, S. e Koutroumbas, K. (2009). Pattern Recognition, 4 edn, Academic Press, San Diego. 